\title{
News Information Platform Optimization Based on the Internet of Things
}

\author{
Hongyun Tan (D) and Yiping Li \\ School of Journalism \& Communication, Jinan University, Guangzhou 510632, China \\ Correspondence should be addressed to Hongyun Tan; thayer99@stu2018.jnu.edu.cn
}

Received 15 April 2021; Revised 11 May 2021; Accepted 19 May 2021; Published 29 May 2021

Academic Editor: Wei Wang

Copyright (C) 2021 Hongyun Tan and Yiping Li. This is an open access article distributed under the Creative Commons Attribution License, which permits unrestricted use, distribution, and reproduction in any medium, provided the original work is properly cited.

\begin{abstract}
The Internet of Things device online recommendation system has been applied in some Internet of Things operating companies and has achieved good results. In the process of designing and implementing the Internet of Things equipment online promotion system, this article uses the news information protocol transmission structure language to explain the use case analysis and activity diagram analysis of the Internet of Things equipment online promotion system and uses the Spring Hibernate (SH) integration framework in the field of the news information topology layer under the Internet of Things. Then, design and implement the technical architecture and main functional modules of the Internet of Things device online recommendation system, effectively improving the development efficiency and operating quality of the Internet of Things device online recommendation system. The system design concept and implementation ideas can be used as a reference for related industries when developing enterprise applications. This article uses the news information protocol transmission structure language to explain the demand analysis of the Internet of Things equipment online promotion system and mainly discusses the analysis of the use case of the Internet of Things equipment online promotion system and the analysis of core business activity diagrams. We completed the design of the Internet of Things device online promotion system based on the news information topology layer platform under the Internet of Things, mainly using the integration framework in the field of the news information topology layer under the Internet of Things to design the technical architecture of the Internet of Things device online promotion system, and design the Internet of Things of the function module structure and data table structure of the equipment online recommendation system. At the same time, they complete the realization of the main functions of the Internet of Things device online recommendation system; elaborate on the realization process of core modules such as Internet of Things device category management, Internet of Things device information management, announcement management, and device recommender management; and discuss the system testing process and application effects.
\end{abstract}

\section{Introduction}

With the development of the news information industry, the volume of news information business continues to grow rapidly. Therefore, the national news communication application market and the development of the Internet of Things are also very rapid. There are now more than 300 million news information users in the world, and there are also millions of Internet users. Experts predict that in recent years, the number of users applying network technology and Internet of Things user technology will continue and rapidly increase. It can be said that these two technologies will greatly change our lifestyle in the next century. News users in the destination country are a very large potential IoT user market, and the development of national news data services has a good foundation. Traditional news and information dissemination channels (such as newspapers, television, and radio) can no longer satisfy people. The demand for information updates has changed with the popularization of the Internet and the emergence of news information management platforms.

As a global strategic emerging industry, the Internet of Things is highly valued by the country and society. The Internet of Things technology uses the advantages of a new 
generation of information technology and communication technology to deeply integrate with traditional industries and promote the revolutionary transformation of traditional industries. The design meets the requirements of national industrial development. Information processing measures will promote the prosperity of the information service industry and achieve the intelligence of the information service industry [1]. With the declining investment cost and the increasing maturity of the two basic conditions of communication technology advancement, the advantage of the Internet of Things to replace people with things can be extended to those scenarios that are limited by time and space. In industries such as logistics and express delivery, transportation, commerce, and retail, the Internet of Things gradually has a certain scale of layout [2]. Even though news communication can keep in touch with the outside world anytime and anywhere, users usually use voice services, while news data services have not been widely used. The commonly used short message service (SMS) only allows to send about 160 bytes of simple information, such a narrow bandwidth is not conducive to Internet access [3]. In order to connect news equipment and the Internet to realize the Internet of Things user data service, people have proposed many solutions, one of which is the Internet of Things user application protocol (news information) [4]. At present, the national news communication is in the development stage of $4 \mathrm{G}$, and then it will be the 5G era [5]. Graphics-based information can be provided in the $3 \mathrm{G}$ environment. For example, news information, mobile games, and other services are developing rapidly, mobile TV services are starting to take off, news industry applications for all walks of life in the society are beginning to rise, and the Internet of Things news value-added information services are becoming a fast stage of development; multimedia-based information can be provided in the $4 \mathrm{G}$ environment, including video phones, video mailboxes, IP phones, video conferences, video streaming media, and online games, among which video services will be the leading application of $4 \mathrm{G}$ networks [6]. The wide application of Internet of Things user network technology has laid a solid foundation for the Internet of Things news value-added information telecommunications business [7].

This article introduces the business types of IoT news value-added information services. In order to realize these services, the protocol transmission structure of the news information platform for IoT users is given on this basis. It further introduces the three components of the logical structure of the IoT user news information platform: access interface layer, function scheduling layer, and business interface layer, and analyzes the role of these three parts in the platform. Finally, the network topology diagram of the news information platform for Internet of Things users is given. As an IoT device operating company, it should vigorously promote IoT devices and use advanced software development technology to build an online recommendation system for IoT devices. Through this recommendation system, increase the promotion of the IoT product market and deliver IoT products and technologies. The news information topology layer under the Internet of Things is a set of mature technical architecture, which provides an excellent mecha- nism for the establishment of scalable, flexible, and maintainable enterprise applications, which can simplify and optimize the development and deployment of application systems. The platform integrates a variety of advanced IT technologies such as database, Internet, and telecommunications; discusses WAP technology and XML technology; and uses the MVC architecture model to implement the WAP website through the WML language. Among them, the Internet of Things news information is based on the multiple frameworks of the news information topology layer platform under the Internet of Things, including the technical framework of the database persistence layer, which is conducive to the development of application systems based on the news information topology layer specification under the Internet of Things; news information protocol transmission structure is incorporated with new ideas, new methods, and new technologies in the field of software engineering; to achieve this effect, designing and implementing a news information management platform have very important economic and social benefits; it is suitable for describing any type of system with object-oriented technology and is especially suitable for requirement specification description. Therefore, this topic uses the news information topology layer and news information protocol transmission structure technology under the Internet of Things to study the construction process of the Internet of Things equipment online promotion system.

\section{Related Work}

Domestic research on IoT news big data started late, and the research content is relatively small, and the coverage is relatively narrow. Especially in the field of IoT news big data news, domestic research focuses on overcoming technical difficulties, and there is even less research on the resulting information sharing and privacy issues. Aazam et al. [8] pointed out in their paper that in the era of big data news in the Internet of Things, the news and health industry will generate a large amount of data every year, and the combination of news and information technology will become closer and closer. Zhang and Wen [9] pointed out that the development of IoT news big data technology in the field of news will become deeper and more extensive. However, problems such as inconsistent data standards, difficulty in data sharing, patient privacy protection, and information security will also arise. The research of Wang and Cai [10] showed that a huge challenge brought by the big data era of the Internet of Things news is the issue of personal privacy protection. People will leave data footprints when they use the Internet. These data footprints are related to each other, leaving a person behind. When the data footprint is large enough, criminals can discover personal private information by integrating and analyzing these data. Talari et al. [11] said that big data of Internet of Things news provides strong data support for clinical research, biotechnology development, and personalized treatment of patients, but medical ethics and data privacy must be considered. They believe that in order to balance the benefits and risks brought by technological innovation, relevant departments must follow the basic principles of the use of IoT news big data, use more advanced 
network security technologies, and establish and improve laws and regulations. Sisinni et al. [12] pointed out in their research that it is necessary to learn from the US and EU's protection models for the privacy information of patients with news Internet of Things news big data, combined with actual situation, and carry out hierarchical and classified protection on the basis of a clear hierarchical structure. Swan [13] pointed out that the national news information news Internet of Things news big data resources need to be further explored and utilized. Current research focuses on theory and experiment and needs to be transformed into practice and application. The theories and methods used in this article have been fully used in other fields. Wang et al. [14] said that based on signal transmission theory and communication privacy management theory, it is concluded that perceived privacy risk has a significant impact on consumers' willingness to provide personal information, and the structural equation model is used to analyze consumers' willingness to participate in online group buying. There are also researchers based on the theory of planned behavior and the theory of technology acceptance, using structural equations to analyze the influencing factors of personal Internet of Things news sharing willingness [15]. Some scholars have used the random news platform database to regress and analyze the factors affecting the information dissemination of the Internet of Things [16].

The news IoT application focuses on providing extensive interoperability, allowing IoT devices to be shared across domains, and reusing knowledge available on the web. In order to solve the problem of interoperability of shared IoT devices across domains, it is proposed to implement semantic interoperability between heterogeneous devices in news network technology and intelligent body technology [17]. It achieves a "Global Understanding Environment" (GUE), and you can achieve interoperability between different devices. The Internet of Things needs to be pushed to a more open, interoperable, and collaborative news Internet of Things. From this, it can be clearly seen that the Internet of Things will gradually form the trend of the news Internet of Things. The prototype news matcher of the news Internet of Things supports news Web technology and implements standard and nonstandard reasoning tasks and a knowledge base of moderate expression. The reason why the news IoT prototype mobile matcher is proposed is because of the architecture and performance of the news IoT [18]. It is impractical to use available inference engines to process news-based information in popular computing scenarios. Scholars put forward the concept of Wisdom Web of Things (W2T), which is an extension of the Wisdom Web in the era of the Internet of Things. The meaning of "wisdom" is that every object in the Internet of Things can know for itself, and other objects can provide the right services to the right objects at the right time and under the right conditions [19]. W2T emphasizes the data cycle, that is, from data to data, from data to information, from information to knowledge, from knowledge to wisdom, from wisdom to service, from service to human beings, and then back to things. The establishment of a smart Internet of Things environment with ubiquitous computing that connects things to things has given enlight- enment to the proposal of the news Internet of Things. Some scholars have proposed the news Internet of Things search engine (WOTS2E), which is a SWoT search engine, based on web crawlers, that can discover associated data endpoints, and use them to enable WoT-enabled devices and services [20-23]. The demand for data generated by equipment and services in the real world has led to the emergence of the Internet of News Things. News Internet of Things is an emerging network that combines the news network and the Internet of Things (IoT/WoT). Its purpose is to associate news-rich and easily accessible information with objects in the real world. The news Internet of Things brings together the News Network and the Internet of Things and links the information marked in news with the physical devices, services, and data that support the Web to achieve seamless data integration. To better understand real-world information, a standardized, scalable, and flexible way is needed to enable real-time Web-connected embedded devices to be discovered globally [24-26].

\section{System Architecture Based on the News Information Platform of the Internet of Things}

3.1. Hierarchical Distribution of News Information under the Internet of Things. The Internet of Things system can be divided into three levels: sensing layer, network layer, and application layer. The sensing layer is used to solve data acquisition problems. It consists of various sensors (temperature and humidity sensors, RFID tags, light intensity sensors, etc.) and sensor gateway constitutes; its core technology mainly includes sensor technology, radio frequency identification technology, Internet of Things user network networking technology (Zigbee network), and fieldbus control (Fieldbus Control System) technology; network layer, also known as the transmission layer, solves the problem of remote transmission of data obtained by the perception layer and integrates sensor networks, news communication technology, and Internet of Things technology [27-29]; the application layer is mainly responsible for information processing and friendly man-machine interface in the Internet of Things system and processing the bottom layer. The incoming data meets their own business needs, and the data resource database is updated in real time to provide unified shared resource support for various businesses and ultimately meet the applications of various industries and fields of the Internet of Things. The wireless news information platform is connected with the short message dispatch center through the standard SMPP protocol or the standard CMPP protocol (Monternet protocol) and can be connected with the short message dispatch center supporting the SMPP/CMPP protocol. According to the concept and characteristics of the Internet of Things, the Internet of Things can be divided into a sensor layer, a network layer, and an application layer according to a bottom-up process.

An information fitting regression classification model is usually applied to binary classification tasks; that is, for each independent variable $X$ in the model, the value of the 
dependent variable $y$ corresponding to it has two cases, namely, 0 and 1 . The information fitting regression model can solve the binary classification task well and has high accuracy and precision. In order to characterize the timeliness of information, this paper introduces the concept of mathematical expectation of timeliness of information (abbreviated as expectation of timeliness), which represents the lifetime of information, that is, the time period during which information truly reflects the state of the source. Given a set of possible messages $A=(1,2, \cdots, n)$ and its probability distribution is $P$, let $f$ denote the probability that message $i$ keeps the information valid in the time interval of $0-t$ function, referred to as the aging probability function. Then, the time-sensitive amount $C$ of message $i$ can be expressed as

$$
C=\left(x_{1}, y_{1}\right),\left(x_{2}, y_{2}\right), \cdots,\left(x_{n}, y_{n}\right)
$$

Formula (1) is the expression of expectation of ageing when the ageing probability function is continuous. The time-effect probability function is the time-effect expectation expression of the discrete case, which can be expressed as follows: it is given the set of possible messages $A-a$, and its probability distribution is $P$. The time point sequence $T$ of the first change of message $i-a$ is given in minutes. Let the timeliness probability distribution of the first change of message $i-a$ be corresponding to $T-i$ :

$$
p\left(y \mid x_{1}, \cdots, x_{n}\right)=\frac{1}{1+e^{-a_{1} \times x_{n}}} .
$$

Therefore, this paper adopts the information fitting regression model as a model to analyze the public's willingness to share news data. Information fitting regression belongs to supervised learning, so it is necessary to use labeled data as the training set to train the function. The training set is a set of training data composed of the features and labels of the data:

$$
p(i)=\frac{e^{-\left(a+b_{1} \times x_{1}+\cdots+b_{i} \times x_{i}\right)}}{e^{a+b i+x i}} .
$$

Among them, $x$ is an $m$-dimensional vector, $Y$ takes a value in $\{0,1\}$, and the classification task is simplified to how to find a decision function $Y$, so that it can have good enough performance on an unknown data set and make a more accurate prediction. In this paper, the information fitting regression model is a regression model of binary variables. The willingness to use dependent variables is a binary variable, and the influencing factors of independent variables are classified data. The dependent variable sharing willingness $Y$ takes the values 0 and 1 , and the independent variable influencing factors are set to $x_{1}, x_{2}, \cdots, x_{n}$, where the value of $Y$ indicates a strong willingness to share and a value of 0 indicates a weak willingness to share. Then, the information fitting function is

$$
1-p(n)=\frac{1}{1+e^{a+b_{1} \times x_{1}+\cdots+b_{n} \times x_{n}}} .
$$

The conditional probability that the $f$ sample has a strong willingness to share is denoted as $P$, and then it can be transformed from equation (4) into

$$
\frac{p(i)}{1-p(i)}=e^{a+b_{i} \times x_{i}}
$$

From equation (5), we can see that $p$ - $i$ is a nonlinear function composed of independent variables, which can be transformed into a linear function through a certain transformation. Define events with weak willingness to share; that is, when $y$ is set to 0 , there are

$$
\ln (p(i))=a+b_{1} \times x_{1}+\cdots+b_{i} \times x_{i}
$$

Calculate the ratio of the probability of a strong willingness to share and the probability of a weak willingness to share:

$$
w(x, y)=\{F(x, y), G(x, y)\}
$$

The ratio of the probability of an event occurring to the probability of not occurring is called the odds. When odds $>1$, it indicates that the situation with strong willingness to use is more likely to occur, and when $\mathrm{O}<$ odds $<1$, it indicates that the situation with weak willingness to use is more likely to occur. Take the natural logarithm of odds to convert it into a linear function:

$$
\begin{gathered}
F(i)=\left\{f_{1}, f_{2}, \cdots, f_{n}\right\}, \\
G(i)=\left\{g_{1}, g_{2}, \cdots, g_{n}\right\}
\end{gathered}
$$

Therefore, equation (8) is also called the functional form or logit form of the information fitting model. For modeling and analysis of dichotomous dependent variables, information fitting regression models have become a common modeling method. Through a large amount of literature reading and analysis, it is found that the information fitting regression model can perform well in the modeling and analysis of individual characteristic factors and study the public's willingness to use the news IoT news big data platform.

3.2. Linear Topology Module Based on IoT Platform. The linear framework based on the Internet of Things platform is a framework that is different from the previous application system research and development. It includes a series of components that can simplify and optimize the research and development, installation, and operation of enterprise applications, thereby improving portability, security, and reuse value. The news information topology layer under the Internet of Things adopts a multilayer distributed application model. At the client layer, the components on the client computer that access the enterprise application, the interface logic between the user and the enterprise application, use the http protocol to access and browse the application server. The presentation layer, a component running on the application server of the news information topology layer under the Internet of Things, interacts with the business logic layer to 
output the business data required by the user in a suitable method. The business logic layer, like the presentation layer, is also a component running on the application server of the news information topology layer under the Internet of Things. As an important part of the wireless news information platform, the news information platform based on WAP technology focuses on the design and implementation of the WAP news website, the data interface module with mobile operators, and the supporting data release and data collection function modules.

Under the news information topology layer specification under the Internet of Things, programmers can develop enterprise-level application systems according to the technical requirements of the news information topology layer under the Internet of Things and a variety of news information topology layer providers under the Internet of Things jointly abide by the specifications of the news information topology layer before and after the version under the Internet of Things to ensure the unity between the news information topology layer platform and enterprise applications under the various Internet of Things. In other words, enterprise applications based on the news information topology layer architecture under the Internet of Things can basically be installed and run on different application servers, without or only a small amount of code editing, which can greatly improve the portability of enterprise applications. The topology of the IoT news platform is shown in Figure 1. For programmers, they only need to focus on the business logic and architecture design of various enterprise applications.

Internet of Things news information is an open source project provided by the Apache software organization. It provides a model-view-controller (MVC) framework for Java Web enterprise applications, which is especially suitable for the development of large-scale and easy-to-expandable Web enterprise applications. The Internet of Things news information provides a common framework for Web enterprise applications, allowing programmers to focus on how to deal with actual business problems. The submission and delivery of short message information are realized through the bidirectional communication interface with the short message center provided by the standard protocol. With the help of the short message bearing function provided by the short message center system, the transmission of information between the information processing server and the mobile platform is realized, and various information services are provided for users. In addition, the Internet of Things news information framework provides many places for expansion and customization. Enterprise applications can easily expand the framework to better adapt to the actual needs of various users. In order to reduce the coupling of code and improve the efficiency of enterprise application research and development, here is a brief description of a news information topology layer enterprise application research and development strategy under the Internet of Things that integrates the Internet of Things news information framework and the Hibernate framework.

The news information platform based on the Internet of Things is a reusable MVC 2 design composed of a group of cooperating classes, Servlet, and JSP tags. This definition describes that IoT news information is a framework, not a library, but the IoT news information framework also includes a rich tag library and utility classes independent of the framework. The Internet of Things news information is a concrete realization of the JSPModel 2 design specification. The logic algorithm of IoT news information is shown in Figure 2. This part of the properties is related to what is a one-to-one correspondence between the user input items in the page form. In the Internet of Things news information framework, the ActionForm class is used to submit the form information entered by the user to the controller; the JSP page is the main view component in a typical MVC application, including the functions of information display and the controller's solution result display. In addition to the above, the IoT news information framework also provides a powerful IoT news information tag library to assist users in processing view logic and uses ActionForm components to transfer information to the control layer (IoT news information core controller).

3.3. Structure Optimization Based on News Information Protocol Transmission. The basic application steps of the protocol transmission structure are based on the news information platform. It worked by using the Unified Modeling Language for application visualization modeling, that is, to describe and document the project that uses object-oriented methods to develop enterprise applications. The news information protocol transmission structure belongs to the third generation of modeling and specification language, which is a method for describing, visualizing, and establishing object-oriented enterprise applications in the enterprise application research and development stage. As a type of modeling language, the news information protocol transmission structure enables software project developers to concentrate on creating the software model and technical structure instead of considering which language and algorithm to choose to complete. After the software model is created, the model can be automatically converted into the selected programming language code by the integrated development tool of the news information protocol transmission structure.

As a subset of the main platform, the news information logical substructure is mainly used to mark and explain the Internet information and user interface sent and received by the news information and news terminal, enabling developers to define the user interface of the news information application in a device-independent manner. Moreover, in most cases, news information uses scripting languages such as WMLScript to directly process warnings and other messages on the news terminal to avoid data interaction between the news terminal and the remote server, thereby reducing the consumption of bandwidth resources. Figure 3 shows the hierarchical structure of the news information platform under the Internet of Things. The news information gateway is the news information agency. The user layer means that the system uses users and system administrators to manipulate the underlying data through a graphical interface. This form not only allows users to operate and edit but also ensures that users do not directly manipulate data packaging data through such an interface isolation method. The news 


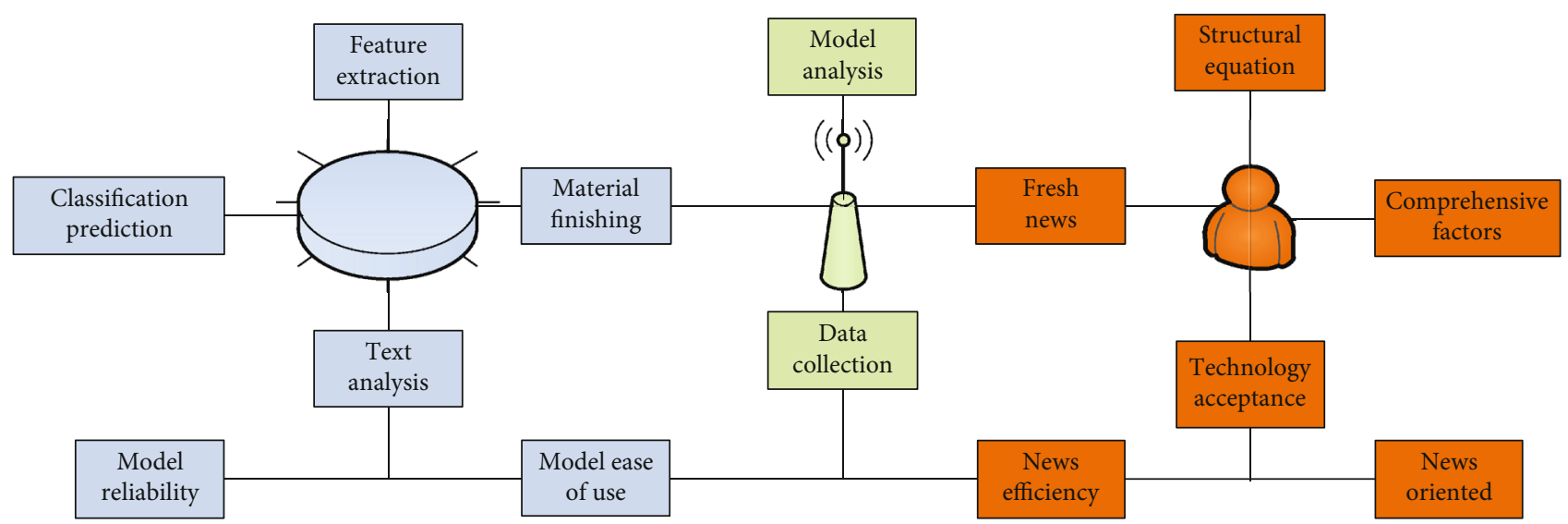

FIGURE 1: Topology structure of IoT news platform.

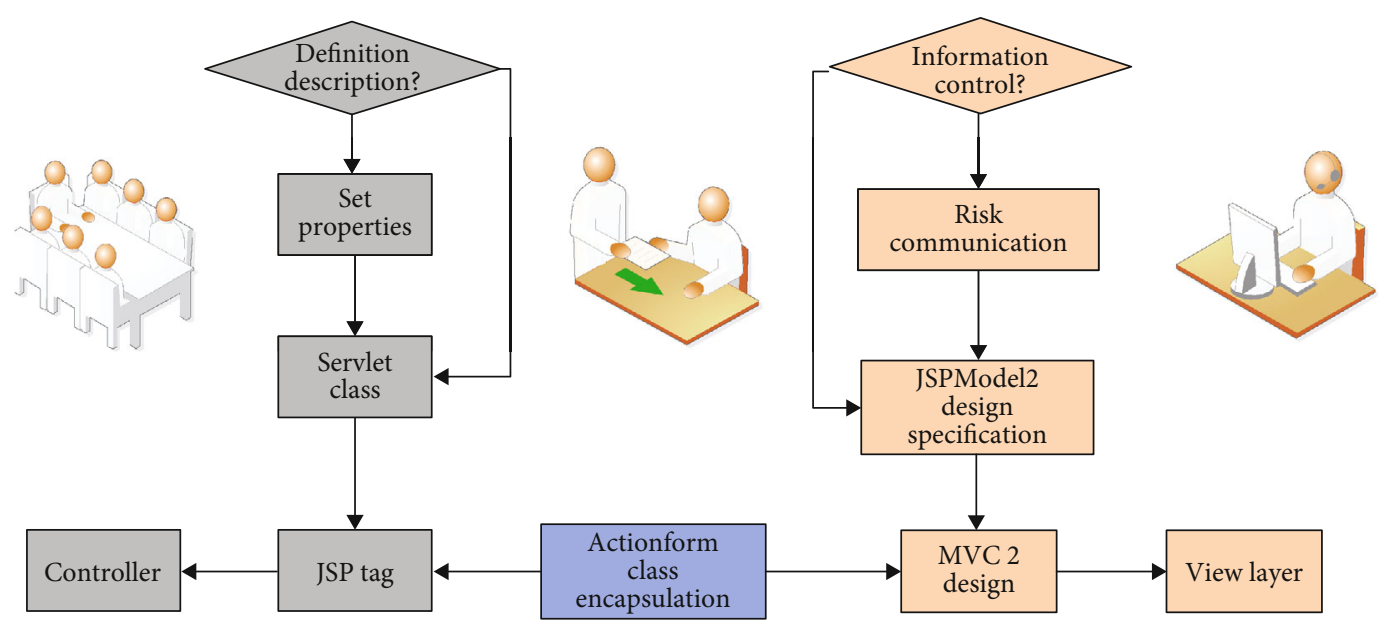

FIGURE 2: The logic algorithm of IoT news information.

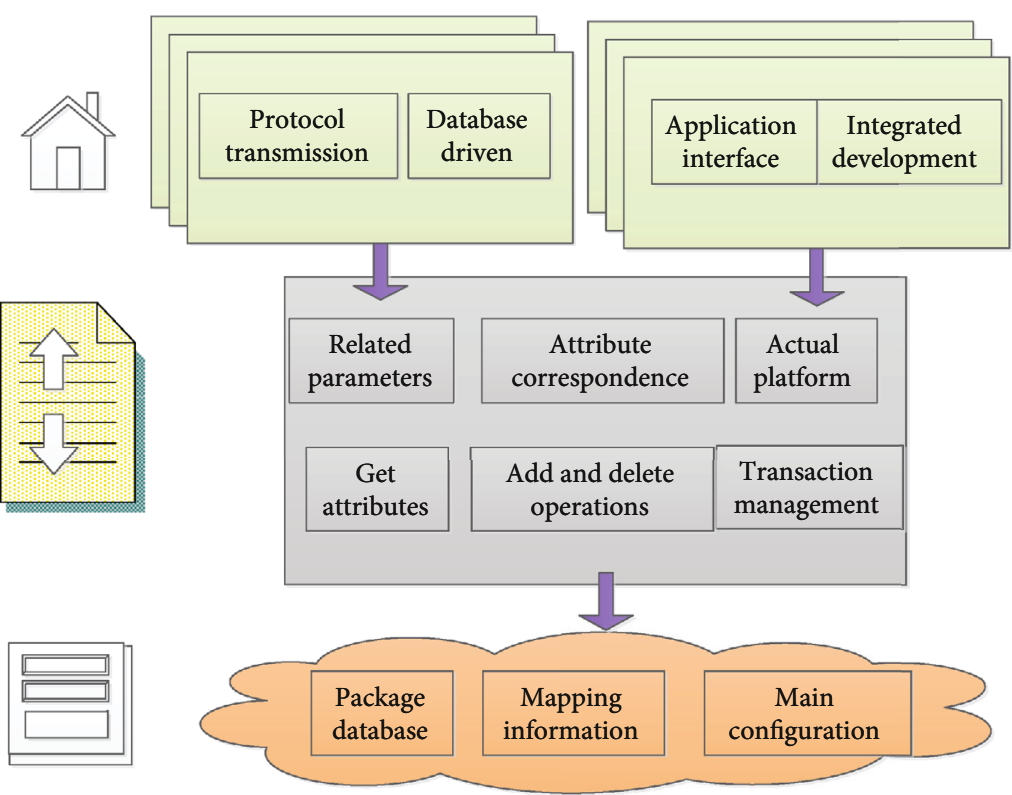

Figure 3: Hierarchical structure of news information platform under the Internet of Things. 
information gateway is used as the gateway between the Internet and the news network to realize the conversion between the Internet protocol and the news information protocol. The news information agency also provides an information content code, which can compress and encode news information data, thereby reducing network data traffic and maximizing the use of the slow data transmission rate of current Internet of Things users. In addition, the news information agent also uses error correction technology to ensure that news information communications will not be seriously affected by changes in the quality of the Internet of Things users' telecommunication lines during network browsing and data transmission.

\section{Design and Implementation of News Information Platform Based on the Internet of Things}

4.1. System Function Module Simulation. System realization is the process of specifically writing information system function modules according to the predesign, which mainly includes the coding of the function modules, the installation and debugging of the Internet of Things equipment online recommendation system, and the testing of the system. The Internet of Things device online recommendation system is an information system developed in a specific operating environment. The Internet of Things device online promotion system is built on the Struts-Hibernate integration framework of the news information topology layer under the Internet of Things, the development tool is Eclipse, and the plugin myeclipse is installed. It can be installed, debugged, configured, and run on Windows, Unix, Linux, and other operating systems, using this part of the tools and operating environment to develop and deploy the Internet of Things device online promotion system, which can be used in local area networks, metropolitan area networks, wide area networks, and other Internet of things In the network environment where the equipment online promotion agency is located. The mapping between the sale information tables is able to draw and describe the correspondence between the entity objects of the Internet of Things device online recommendation system and the data table. Table 1 shows the data table of the IoT news entity object.

From the perspective of the overall test effect of the model, the model setting is relatively reasonable and the overall fitting effect is good. The prediction accuracy rate of the model reached $79.52 \%$. From further analysis of the regression results, we can see that the status of news information dissemination, the time of exposure to the Internet of Things, the experience of using the Internet to transmit news information, and the habit of recording body data, the signs of the coefficients of these variables are consistent with the actual situation, and they are all consistent with the actual situation. Then, they passed the empirical analysis test based on individual characteristic factors at a significance level of $10 \%$. The regression coefficient of the Internet of Things is 0.5806 , and the $P$ value is 0.073 . At a significance level of 0.1 , the Internet of Things has a positive effect on the willingness to
TABle 1: Data table of IoT news entity object.

\begin{tabular}{lccc}
\hline Sample attributes & Scale range & Distribution number & Rate \\
\hline Sample 1 & A1 & 99 & 0.31 \\
Sample 2 & A2 & 122 & 0.21 \\
Sample 3 & A3 & 82 & 0.08 \\
Sample 4 & A4 & 45 & 0.4 \\
\hline
\end{tabular}

share news information. That is, IoT users are more willing to share their news data than non-IoT users. The reason may be that IoT users have a higher risk appetite than nonIoT users and are more willing to try new things. The regression coefficients of bachelor's degree and master's degree and above in the educational level are 0.7450 and 1.0486 , respectively, and the corresponding $P$ values are 0.032 and 0 , respectively. They are both less than the significance level of 0.05 . The degree of education has a positive effect on the willingness to share news information; that is, respondents with a bachelor degree or above are more willing to share news information. In the status of body news information, the regression coefficients for scoring one's body into 3 and 5 points are 3.3122 and 3.6256, respectively, and the corresponding $P$ values are 0.004 and 0 , respectively. Figure 4 shows the sharing of news information based on regression coefficients. At a significance level of 0.01 , it has a positive effect on the willingness to share news information; that is, respondents with better physical conditions are more willing to share news information than those with poorer physical conditions.

In the item of time of exposure to the Internet of Things, the regression coefficient of exposure to the Internet of Things for more than ten years is 1.3135 , and the $P$ value is 0.016 . At a significance level of 0.05 , it has a positive effect on the willingness to share news information. The news section correspondence table is used to record each news and its corresponding section node. The same news can correspond to multiple section nodes at the same level, but in general, a news item corresponds to only one section node. The main information includes news number and section number. That is, respondents who have been exposed to the Internet for a longer time are more willing to share news information on the Internet of Things. In the experience of using the network to transmit news information, the regression coefficient is 0.6282 , and the $P$ value is 0.053 ; at a significance level of 0.1 , it has a positive effect on the willingness to share news information; that is, respondents who have experience of delivering news information online for medical treatment are more willing to share news information on the Internet of Things. In the item of whether there is a habit of using news equipment to record body information, the regression coefficient is 0.8576 , and the $P$ value is 0.011 . Figure 5 shows the distribution of the significance level test for different sample groups. At the significance level of 0.5 , it has a positive effect on the willingness to share news information; that is, respondents who have the habit of recording physical data are more willing to share news information.

The path coefficient from perceived ease of use to perceived usefulness is 0.80 , indicating that perceived ease of 


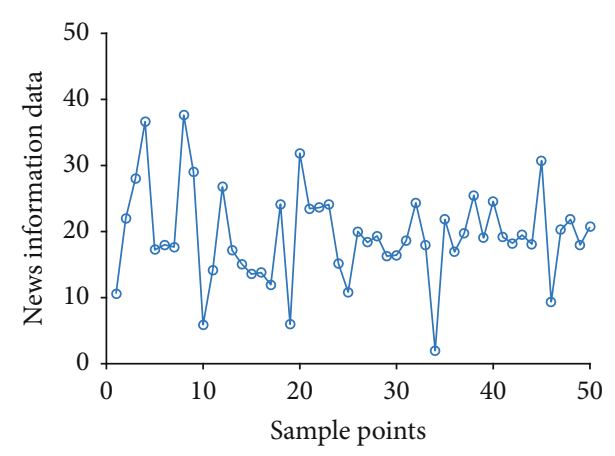

Figure 4: News information sharing based on regression coefficients.
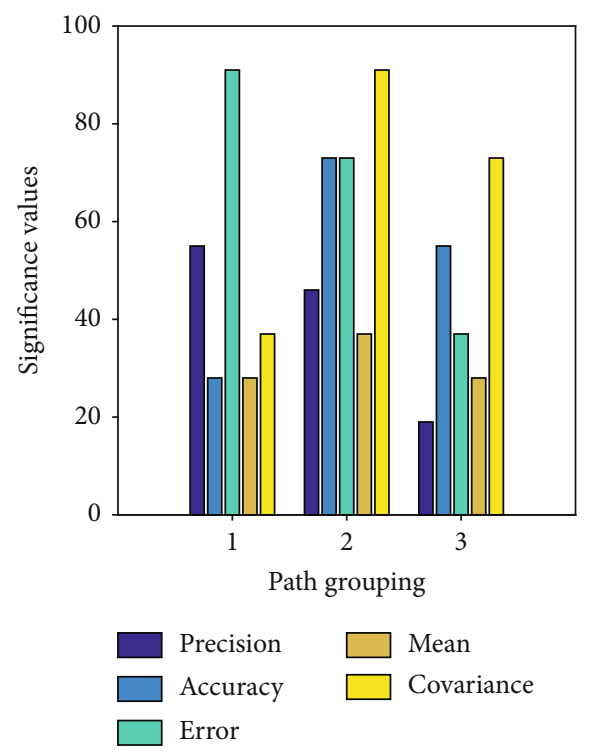

Figure 5: The distribution of the significance level of different sample groups.

use has a significant positive effect on the public's perceived usefulness of news information sharing, which is consistent with the technology acceptance model proposed by Davis. Respondents believe that news information sharing is a useful technology; the premise is that news information sharing is easy to realize and use. The path coefficient from perceived ease of use to willingness to share is 0.05 , indicating that the perceived ease of use has a positive effect on the public's willingness to share news information using the news IoT news big data platform, but it is not significant. The path coefficient from perceived usefulness to sharing willingness is 0 . 04. It shows that the perceived usefulness has a positive effect on the willingness to use the news big data platform to share news information, but it is not significant. The path coefficient from perceived risk of willingness to share is 0.14 , indicating that the perceived risk has a negative impact on the public's willingness to share news information. The working method of the news information management platform developed in this paper adopts the dynamic web page management method, which is developed after solving the various shortcomings of the static state. In general, the results of structural equation analysis are consistent with the assumptions.

4.2. Entity News Platform Database Design. The physical news platform database is a single classifier, which has a bottleneck in performance improvement. News information integrated learning, that is, the random news platform database algorithm, is to gather a single entity news platform database and determine the category of the sample to be classified by collecting statistics on the classification results of each entity news platform database. It is equivalent to that each classifier will have a classification result. There are two important randomizations in the Random-News platform database, as shown in Figure 6, which is the training efficiency of the news information dataset algorithm. Since the process of randomly extracting training sample sets and feature subsets when constructing each entity news platform database is independent, the overall is the same, and each entity news platform database is an independent and identically distributed sequence of random variables. Therefore, the training of the random news platform database can be realized through parallel processing, which ensures the efficiency and scalability of the random news platform database algorithm.

First, use the train test split in the library to randomly divide all data into training subsets and test subsets. The train test split function is a commonly used function in crossvalidation. The function is to randomly select train data and test data from the sample proportionally. The test size (the proportion of samples) selected in this paper is $20 \%$, and the number of random seeds is 2 . In the access type field, a value of " 1 " indicates that the user's access type is viewing and browsing; a value of " 2 " indicates that the user's access type is sharing. There are other new access types that can be added at any time in the future. If $n$ estimators are too small, it is easy to underfit, and if $n$ estimators are too large, it is easy to overfit. Generally, choose a moderate value for the random news platform database, increasing the "number of submodels" ( $n$ estimators) which can significantly reduce the variance of the overall model. The accuracy of the model will increase as the "number of submodels" increases. Since the decrease is the second term of the overall model variance formula, there is an upper limit for the improvement of accuracy. The random state is the seed used by the random number generator.

4.3. System Test and Application Effect. First, average the scores of the four questions in the sharing willingness part of the questionnaire, and classify those with an average score greater than or equal to 4 into one category, which indicates strong willingness to share, and classify those with an average score less than or equal to 2 into one category, indicating the willingness is weak. Putting the average score between 2 and 4 points into the first category indicates that the willingness to share is average. After processing, the respondents were divided into three categories, with the number of persons in each category as shown in Figure 7, which is the statistical data of sample correlation. It can be seen from the figure that 


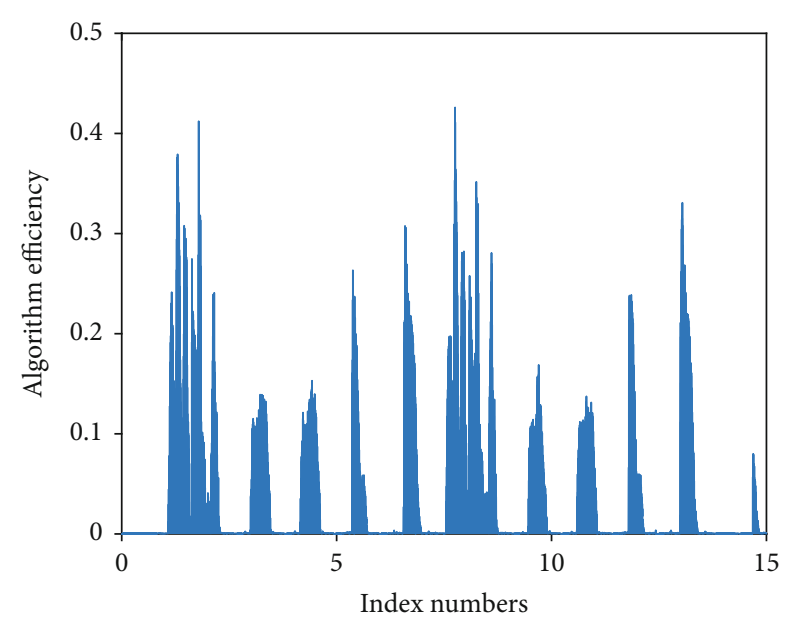

Figure 6: Algorithm training efficiency of news information data set.

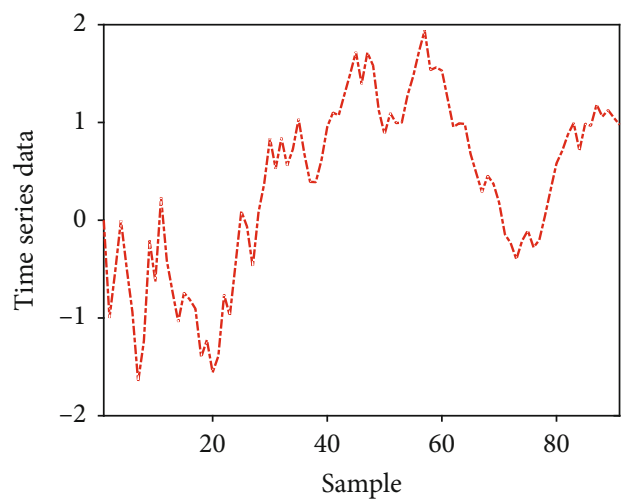

FIgURE 7: Statistical data of sample correlation.

there are 53 respondents with low willingness to share, 122 respondents with general willingness to share, and 154 respondents with strong willingness to share. The number of the three types of respondents is quite different, and the distribution is not very uniform, which poses a certain challenge to the subsequent classification. It can be seen from the figure that the distribution of the remaining six variables is not uniform, except for the number of Internet of Things user surveyed, non-IoT user surveyed, and the survey's habits of recording body data. By drawing a heat map of the correlation between the eight variables, it can be seen that the correlation between these eight variables is mostly in the normal range. The following result indicates that the correlation is not obvious, which also poses a challenge to the subsequent classification.

CMIN/DF is called relative chi-square or normalized chisquare. It is the minimum sample difference divided by the degree of freedom. Generally, this value is allowed to reach 5 as an appropriate fit. The relative chi-square value in this model is 3.786, which is less than 5, which meets the requirements, so this model is acceptable. RMSEA is the root mean square of the approximate error, and its value is less than or equal to 0.05 which means that the model fits very well, and its value is less than 0.08 , which means that the model fits

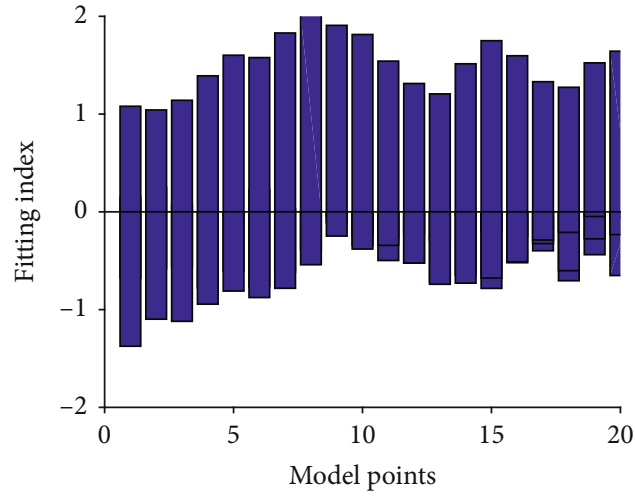

Figure 8: Histogram of IoT model fitting index.

properly. The root mean square of the approximate error in this model is 0.092 , which is greater than 0.08 , which does not meet the requirements, so the model should be rejected. GFI is the goodness of fit index, and the range is between 0 and 1. To accept the model, GFI should be greater than or equal to 0.90. The goodness of fit index of this model is 0.911 , which is greater than 0.90 , which meets the requirements, so this model is acceptable. IFI is a value-added fitting index. The range of its value is not guaranteed to be between 0 and 1 . Figure 8 shows the histogram of the fit index of the IoT model. IFI close to 1 indicates a good fit, and it is greater than 0.90 which indicates an acceptable fit. The value-added fitting index of this model is 0.933 , which is greater than 0.9 . It meets the requirements, so this model is acceptable. The crawler module is a core module for the system to search for news, so it is important to ensure that this module can run correctly. The picture shows the functional test description of the crawler module and the specific display of the use cases. CFI is a comparative fitting index. A value close to 1 indicates a good fit, and a value greater than 0.90 indicates an acceptable fit.

The comparative fitting index of this model is 0.936 , which is greater than 0.90 . It meets the requirements, so this model is acceptable. TLI is the Tucker-Lewis coefficient, and the range of its value is not guaranteed to be between 0 and 1 . TLI close to 1 indicates a good fit, and it is greater than 0.90 which means that the fit is acceptable. The TLI of this model is 0.919 , which is greater than 0.90 . The empirical analysis is based on individual subjective factors in the requirements, so this model is acceptable. AGFI is an adjusted goodness-of-fit index, and the range of variation is between 0 and 1 , which is greater than 0.50 which means that the fit is acceptable. The adjusted goodness of fit index of this model is 0.655 , it is greater than 0.5 which means it meets the requirements, so this model is acceptable. It can be seen from the above indicators that although the value of RMSEA is not in the ideal range and is slightly larger than the maximum value, the rest of the fitting indices are in the ideal range and meet the requirements.

The accuracy of classification is calculated by the predicted classification of the test data set and the actual classification of 300,000 square meters of data. It is based on the empirical analysis of individual characteristic factors. For example, when a certain data belongs to the first category, 


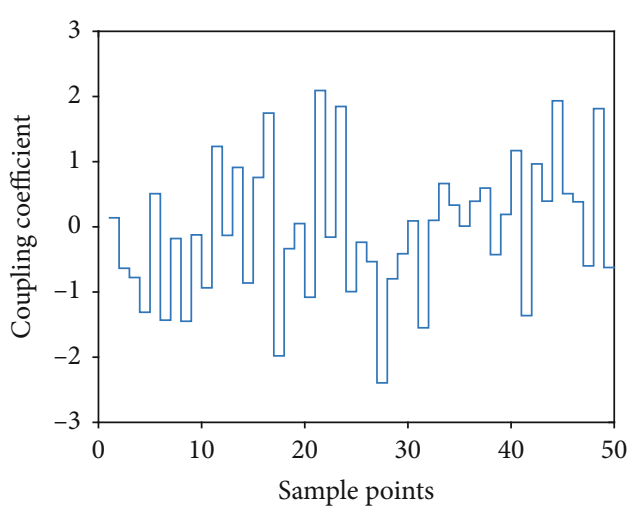

FIgure 9: Distribution of news information data coupling factor.

the program divides it into the second or third category which is the classification error, and the correct rate of the predicted classification is obtained by calculating the proportion of the correct number of classifications to the total number. Through the calculation of the program, on this data set, the accuracy rate of the random news platform database model classification is 0.455 , which exceeds 0.333 , indicating that the classification has a certain effect, but it is still not very prominent. Eventually, 3 of the 12 samples of the first category were classified into the first category, 5 were incorrectly classified into the second category, and 4 were incorrectly classified into the third category; out of the 20 samples of the second category, 16 of them were finally classified in the second category, and 4 samples were mistakenly classified into the third category; finally, 11 of the 34 samples in the third category were classified into the third category, 1 was incorrectly classified into the first category, and 22 were mistakenly classified into the second category. Figure 9 shows the distribution of news information data coupling factors. Therefore, the second type is easier to distinguish, with higher accuracy, and the first and third types have lower accuracy and higher error rate. It is more intelligent, and the timeliness has also been greatly improved, which greatly improves the work efficient, and can provide a more intelligent site search function, a better user experience.

\section{Conclusion}

The main design goal of the Internet of Things user news information platform is to achieve a platform that makes full use of the information resources of traditional media and realizes multiple Internet of Things news value-added information services including SMS, MMS, and news information. This paper will focus on the design ideas of a news information platform for Internet of Things users based on news information technology and take news information as an example to study how to implement a news information platform based on news information technology under the GPRS network built by operators. The news information platform is based on news information technology, as an important part of the news information platform for Internet of Things users, it focuses on the design of news information news websites and data interface modules with news operators, and it supports data release and data collection function modules.
With the implemented solutions, the platform integrates a variety of advanced IT technologies such as database, Internet of Things, and telecommunications, discusses news information technology, and uses architecture pattern language to implement a news information website. The significance level supports the null hypothesis, and the two variables of age and occupation cannot pass the test. At the same time, the prediction accuracy of the model is $79.52 \%$, indicating that the model has a better fitting effect. At the same time, the random news platform database classification method is used to analyze the relationship between individual characteristics and the willingness to share. In summary, the development of this article focuses on user experience improvement and adopts a dynamic web management method to achieve uninterrupted service to users when news information is released. The platform has good functional scalability and builds a system architecture with strong subsequent scalability. The design and implementation of this article have been directly applied in the project, and also provide a research foundation and certain practical experience for further expanding the value-added services of the Internet of Things news and significantly expand the functions of the value-added information service platform system of the Internet of Things news.

\section{Data Availability}

The data used to support the findings of this study are available from the corresponding author upon request.

\section{Conflicts of Interest}

The authors declare that they have no known competing financial interests or personal relationships that could have appeared to influence the work reported in this paper.

\section{Acknowledgments}

This work was supported by Jinan University.

\section{References}

[1] C. Tao and L. Xiang, "Municipal solid waste recycle management information platform based on internet of things technology," Multimedia Information Networking and Security, vol. 3, pp. 729-732, 2019.

[2] H. C. Y. Chan, "Internet of things business models," Journal of Service Science and Management, vol. 8, no. 4, p. 552, 2019.

[3] J. Ye, B. Chen, Q. Liu, and F. Yu, "A precision agriculture management system based on Internet of Things and WebGIS," IEEE Geoinformatics, vol. 7, pp. 1-5, 2020.

[4] J. Lee, S. H. Kim, S. B. Lee, H. J. Choi, and J. J. Jung, "A study on the necessity and construction plan of the internet of things platform for smart agriculture," Journal of Korea multimedia society, vol. 17, no. 11, pp. 1313-1324, 2019.

[5] A. Bahga and V. K. Madisetti, "Blockchain platform for industrial internet of things," Journal of Software Engineering and Applications, vol. 9, no. 10, pp. 533-546, 2018.

[6] J. Mineraud, O. Mazhelis, and X. T. Tarkoma, "A gap analysis of Internet-ofings platforms," Computer Communications, vol. 89, pp. 5-16, 2018. 
[7] Z. D. R. Gnimpieba, A. Nait-Sidi-Moh, D. Durand, and J. Fortin, "Using Internet of Things technologies for a collaborative supply chain: application to tracking of pallets and containers," Procedia Computer Science, vol. 56, pp. 550-557, 2020.

[8] M. Aazam, I. Khan, A. A. Alsaffar, and E. N. Huh, "Cloud of things: integrating Internet of Things and cloud computing and the issues involved," Applied Sciences \& Technology, vol. 5, pp. 414-419, 2018.

[9] Y. Zhang and J. Wen, "The IoT electric business model: using blockchain technology for the internet of things," Peer-to-Peer Networking and Applications, vol. 10, no. 4, pp. 983-994, 2017.

[10] K. Wang and K. Cai, "Design of field information monitoring platform based on the internet of things," Internet of Things, vol. 312, pp. 597-602, 2019.

[11] S. Talari, M. Shafie-khah, P. Siano, V. Loia, A. Tommasetti, and J. Catalão, "A review of smart cities based on the internet of things concept," Energies, vol. 10, no. 4, p. 421, 2017.

[12] E. Sisinni, A. Saifullah, S. Han, U. Jennehag, and M. Gidlund, "Industrial internet of things: challenges, opportunities, and directions," IEEE Transactions on Industrial Informatics, vol. 14, no. 11, pp. 4724-4734, 2018.

[13] M. Swan, "Sensor mania! The internet of things, wearable computing, objective metrics, and the quantified self 2.0," Journal of Sensor and Actuator Networks, vol. 1, no. 3, pp. 217-253, 2019.

[14] P. Wang, S. Chaudhry, and L. Li, "A visualization platform for internet of things in manufacturing applications," Internet Research, vol. 3, pp. 18-23, 2018.

[15] I. Yaqoob, E. Ahmed, I. A. T. Hashem et al., "Internet of things architecture: recent advances, taxonomy, requirements, and open challenges," IEEE Wireless Communications, vol. 24, no. 3, pp. 10-16, 2017.

[16] D. Bandyopadhyay and J. Sen, "Internet of things: applications and challenges in technology and standardization," Wireless Personal Communications, vol. 58, no. 1, pp. 49-69, 2019.

[17] A. J. C. Trappey, C. V. Trappey, U. Hareesh Govindarajan, A. C. Chuang, and J. J. Sun, "A review of essential standards and patent landscapes for the Internet of Things: A key enabler for industry 4.0," Advanced Engineering Informatics, vol. 33, pp. 208-229, 2017.

[18] J. A. Stankovic, "Research directions for the Internet of Things," IEEE Internet of Things Journal, vol. 1, no. 1, pp. 39, 2019.

[19] S. M. R. Islam, D. Kwak, M. D. H. Kabir, M. Hossain, and K. S. Kwak, "The internet of things for health care: a comprehensive survey," IEEE access, vol. 3, pp. 678-708, 2018.

[20] S. Li, T. Tryfonas, L. Li et al., "The Internet of Things: a security point of view," Internet Research, vol. 26, no. 2, pp. 337359, 2016.

[21] M. Cui, J. Lu, A. Zhao, and C. Wei, "Application research of information platform of coal enterprise based on internet of things," Coal Technology, vol. 1, pp. 32-39, 2021.

[22] Y. Lu, Y. Qi, S. Qi et al., "Secure deduplication-based storage systems with resistance to side-channel attacks via fog computing," IEEE Sensors Journal, vol. 1, no. 1, p. 1, 2021.

[23] W. Wang, N. Kumar, J. Chen et al., "Realizing the potential of the Internet of Things for smart tourism with $5 \mathrm{G}$ and AI," IEEE Network, vol. 34, no. 6, pp. 295-301, 2020.

[24] W. Wei, Q. Ke, J. Nowak, M. Korytkowski, R. Scherer, and M. Woźniak, "Accurate and fast URL phishing detector: a con- volutional neural network approach," Computer Networks, vol. 178, article 107275, 2020.

[25] A. Zielonka, A. Sikora, M. Wozniak, W. Wei, Q. Ke, and Z. Bai, "Intelligent Internet of Things system for smart home optimal convection," IEEE Transactions on Industrial Informatics, vol. 17, no. 6, pp. 4308-4317, 2021.

[26] C. S. Yang, "Adoption of software testing in Internet of Things: a systematic literature mapping," Computing Reviews, vol. 61, no. 2, pp. 72-73, 2020.

[27] A. al-Qerem, M. Alauthman, A. Almomani, and B. B. Gupta, "IoT transaction processing through cooperative concurrency control on fog-cloud computing environment," Soft Computing, vol. 24, no. 8, pp. 5695-5711, 2020.

[28] M. Shafiq, Z. Tian, Y. Sun, X. du, and M. Guizani, "Selection of effective machine learning algorithm and Bot-IoT attacks traffic identification for internet of things in smart city," Future Generation Computer Systems, vol. 107, pp. 433-442, 2020.

[29] D. Li, L. Deng, B. Bhooshan Gupta, H. Wang, and C. Choi, "A novel $\mathrm{CNN}$ based security guaranteed image watermarking generation scenario for smart city applications," Information Sciences, vol. 479, pp. 432-447, 2019. 